\title{
Production of yellow-emitting carbon quantum dots from fullerene carbon soot
}

\author{
Qinghong Zhang ${ }^{1,2}$, Xiaofeng Sun ${ }^{1,3}$, Hong Ruan ${ }^{1}$, Keyang Yin ${ }^{1}$ and Hongguang $\operatorname{Li}^{1 *}$
}

\begin{abstract}
Carbon quantum dots (CQDs) have emerged as a new generation of photoluminescent nanomaterials with wide applications. Among the various synthetic routes for CQDs, the acid-refluxing method, which belongs to the group of "top-down" methods, offers the advantage of large-scale production of CQDs and uses cheap and abundantly available starting materials. In this study, we evaluated the potential of fullerene carbon soot (FCS), a by-product obtained during the synthesis of fullerene, as the starting material for CQD production. It was found that FCS can be successfully converted to CQDs in high production yield in mixed acids, i.e., concentrated $\mathrm{HNO}_{3}$ and $\mathrm{H}_{2} \mathrm{SO}_{4}$, under mild conditions. The fluorescence quantum yield $(\Phi)$ of the as-produced CQDs is in the range of $3 \%-5 \%$, which is the highest value for CQDs obtained from "top-down" methods. Importantly, the CQDs prepared by this method show emission in the yellow range of the visible light, which is advantageous for their various potential applications. Further investigations reveal that the CQDs are highly photostable over a wide $\mathrm{pH}$ range and show good resistance against ionic strength and long-term UV irradiation. This further expands their potential use under harsh conditions.
\end{abstract}

Keywords: carbon quantum dots, fullerene, carbon soot, photoluminescent, top-down

\section{INTRODUCTION}

Fluorescence imaging is indispensable in optical and biological studies. Quantum dots (QDs) are considered as great substitutes of conventional organic dyes and genetically engineered fluorescent proteins used in fluorescence imaging of biological samples because of their excellent photostability, sharpe emission bands, and tunable emission wavelengths [1-3]. However, conventional semiconducting QDs contain heavy metal elements, which are harmful to humans when used in biological imaging because of their potential toxicity [4-6]. In 2004, carbon quantum dots (CQDs) were accidentally discovered by $\mathrm{Xu}$ et al. [7] during the purification of single-walled carbon nanotubes (SWNTs), which quickly gained immense attention during the following years owing to their good biocompatibility, high photostability, and tunable photoluminescent properties [8-11].

To fully realize the promising applications of CQDs, their large-scale and low-cost production is necessary. Thus far, various methodologies have been reported for the synthesis of CQDs. These methodologies can be mainly classified into two categories, namely, the "top-down" and "bottom-up" methods. The former includes laser ablation [12-14], plasma treatment [15], electrochemical oxidation [16-19], and acid-refluxing [20-31], while the latter is mainly focused on pyrolysis using simple heating and hydrothermal or microwave treatment [32-35]. Among these methods, the acid-refluxing of raw carbon materials offers a large-scale production of CQDs as it only needs simple instruments and can be easily scaled-up using standard industrial equipment. Theoretically, nearly all carbon-rich resources such as carbon nanotubes (CNTs) [20], carbon fibers [21], activated carbon [22], coals [23-25], petroleum coke [26-28], and carbon soot [29-31] can be used as the starting materials. For the large-scale production of CQDs, CNTs and carbon fibers can be ideal candidates if their prices can be further lowered in the future. Coal is abundant and cheap, and is thus a promising raw material for the synthesis of CQDs by acid reflux. However, the CQDs obtained by using coal as the raw material suffer from both low production yield $(Y)$ and fluorescence quantum yield $(\Phi)$ because of the complicated composition of coal. Carbon soot is regarded as a promising starting material

\footnotetext{
${ }^{1}$ Laboratory of Clean Energy Chemistry and Materials, Lanzhou Institute of Chemical Physics, Chinese Academy of Sciences, Lanzhou 730000, China

${ }^{2}$ China Research Institute of Daily Chemical Industry, Taiyuan 030001, China

${ }^{3}$ University of Chinese Academy of Sciences, Beijing 100049, China

*Corresponding author (email: hgli@licp.cas.cn)
} 
to obtain high-quality CQDs as it is normally obtained by burning and the composition as well as the size distribution of the carbon granules present in it is relatively uniform and the carbon content is usually very high. A limitation is that currently the carbon soot used for CQD synthesis is mainly obtained by burning candles [29], natural gas [30], or tires [31]. Thus, the soot produced is quite limited in quantity and is difficult to be gathered. Hence, finding an alternative source of carbon soot is imperative for large-scale and low-cost production of CQDs.

As one of the earliest discovered carbon nanomaterials, fullerenes have received considerable attention since their discovery owing to their unique structural, optical, and electrical properties. With the smooth progress in the fundamental research on fullerenes their commercialization has also increased. Nowadays, tons of fullerenes are produced every year, thus producing a large amount of carbon soot (>85 wt.\%) as the by-product. It would be of great significance if this soot can be converted into more valuable products. The use of fullerene carbon soot (FCS) in capacitor electrodes or as catalyst supports for the reduction of NO have been reported $[36,37]$. In a recent work, Hu et al. [38] reported that FCS can be used for producing single-walled CNTs in high Y. Herein, we presented a detailed evaluation of the potential of FCS to be used as the starting material for the production of CQDs. It was found that the CQDs obtained from FCS exhibited a high $Y$ and $\Phi$ and had yellow-emitting characteristics. Moreover, they could endure long-term UV irradiation and were stable in solutions with a wide $\mathrm{pH}$ range and high salinity. All of these merits open up a variety of potential applications for CQDs.

\section{EXPERIMENTAL SECTION}

\section{Materials}

The FCS used for the preparation of CQDs was purchased from Suzhou Dade Carbon Nanotechnology Co., Ltd. and Jiangsu XFNANO Materials Tech Co., Ltd. It was obtained as a by-product during the manufacture of fullerenes. Concentrated $\mathrm{HNO}_{3}(65 \%-68 \%)$ and $\mathrm{H}_{2} \mathrm{SO}_{4}(95 \%-98 \%)$ were obtained from Laiyang Kangde Chemicals Co., Ltd. and were used as received. Dialysis bags with varying molecular weight cut-offs were provided by Membrane Filtration Products, Inc. Beijing Thai technology Co., Ltd.

\section{Synthesis of CQDs}

For optimizing the synthetic conditions, concentrated $\mathrm{HNO}_{3}$ and $\mathrm{H}_{2} \mathrm{SO}_{4}$ were pre-mixed by cup sonication (model number: $230 \mathrm{~T}$ ) for $40 \mathrm{~min}$. $50-250 \mathrm{mg}$ FCS was added to this mixture. The resulting mixture was then cup sonicated for $1 \mathrm{~h}$, heated to $80-120^{\circ} \mathrm{C}$ under stirring and allowed to react for $12-36 \mathrm{~h}$. After the reaction mixture was cooled down to room temperature, it was subjected to an ice-water bath and a large amount of water (> 6 times) was added slowly followed by the addition of $\mathrm{K}_{2} \mathrm{CO}_{3}$ to neutralize the excess acids (until $\mathrm{pH}=7$ ). The mixture was then centrifuged (Anke TGL-16G) for $30 \mathrm{~min}$ at a rate of $10,000 \mathrm{r} / \mathrm{min}$ to remove large particles followed by dialysis for 5-7 days with frequent changing of water using a dialysis bag of $500 \mathrm{Da}$ to give the final product.

In a typical experiment for stepwise dialysis, the mixing volume ratio of $\mathrm{HNO}_{3}$ and $\mathrm{H}_{2} \mathrm{SO}_{4}$ was fixed at 1:1 and the total volume was $4 \mathrm{~mL}$. $50 \mathrm{mg}$ of FCS was added. Refluxing was performed at $100^{\circ} \mathrm{C}$ for $36 \mathrm{~h}$. After centrifugation, the sample was first dialyzed with a dialysis bag with a molecular weight cut-off of $100 \mathrm{Da}$ to remove the inorganic salts and tiny fragments present in it. The sample within the bag was then successively subjected to dialysis using the bags with molecular weight cut-offs of 500, 1000, and 2000 Da. During each step in the dialysis process, the dialysate was concentrated to $\sim 5 \mathrm{~mL}$ and the final sample within the dialysis bag had a volume of $\sim 15 \mathrm{~mL}$. The solutions of the as-produced CQDs were concentrated using rotary evaporation or lyophilized wherever necessary.

\section{Characterizations}

Transmission electron microscopy (TEM) images were taken on a JEM-1011 system. High-resolution TEM (HRTEM) images were recorded on a HRTEM JEOL 2100 system operating at $200 \mathrm{kV}$. The specimens were prepared by drop-casting the sample solution onto a carbon-coated copper grid followed by drying at room temperature. $\mathrm{X}$-ray diffraction (XRD) pattern was obtained using a D8 Advance X-ray diffractometer with a wavelength $(\lambda)$ of $0.15418 \mathrm{~nm}$. Raman spectra were recorded on a Renishaw Raman microscope using a 514-nm laser excitation at room temperature. Fourier transform infrared (FTIR) spectra were obtained using an FTIR spectrophotometer (VERTEX-70). UV-vis spectra were obtained by a UV-vis spectrophotometer (U-4100). X-ray photoelectron spectroscopy (XPS) was carried out using an $\mathrm{X}$-ray photoelectron spectrometer (ESCALAB 250) with a monochromatized Al Ka X-ray source $(1486.71 \mathrm{eV})$. Thermogravimetric analysis (TGA) was carried out using a DSC 822e (Piscataway, NJ) under nitrogen with a scanning speed of $5^{\circ} \mathrm{C} \mathrm{min}^{-1}$. Fluorescence spectra were obtained using a spectrofluorometer (LS-55). Elemental analysis was carried out using an organic elemental analyzer (Vario 
EI III). The fluorescence lifetimes and time resolved emission fluorescence spectra of the CQDs were measured on an Edinburgh Instruments FLS920 with a time correlated single photon containing (TCSPC) method. The data were deconvoluted with the instrument response function, recorded using dispersed light, and fitted to a multi-exponential function. The fluorescence quantum yields were measured with a spectrofluorometer (FLSP920, Edinburgh Instruments Ltd.) equipped with an integrating sphere, which had a spherical cavity with an inner diameter of 120 $\mathrm{mm}$. $3 \mathrm{~mL}$ of the sample solution was sealed in a quartz cell $(1 \mathrm{~cm} \times 1 \mathrm{~cm})$ with a plug. The same volume of solvent was used as the blank sample.

\section{RESULTS AND DISCUSSION}

\section{Preparation of CQDs}

To synthesize CQDs, a traditional "top-down" method was adopted. The schematic of the synthetic procedure used in this study is shown in Fig. 1a. In brief, a desired amount of FCS was added to a mixture of concentrated $\mathrm{HNO}_{3}$ and $\mathrm{H}_{2} \mathrm{SO}_{4}$. After the homogenization by sonication, the mixture was subjected to refluxing. It is known that sonication itself can be an effective method to produce nanometer-sized carbon materials such as graphene sheets [39]. Here, however, the purpose of sonication was mainly the homogenization of FCS in the mixed acids, since the particles could be clearly seen as visible black particles prior to the acid refluxing. First, the acid treatment temperature and time were fixed at $100^{\circ} \mathrm{C}$ and $24 \mathrm{~h}$, respectively, and the influence of the $\mathrm{HNO}_{3}: \mathrm{H}_{2} \mathrm{SO}_{4}$ volume ratio $(\rho=$ $\left.V_{\mathrm{HNO} 3} / V_{\mathrm{H} 2 \mathrm{SO} 4}\right)$ and total volume of the mixed acids $\left(V_{\text {total }}=\right.$ $\left.V_{\mathrm{HNO} 3}+V_{\mathrm{H} 2 \mathrm{SO} 4}\right)$ was investigated. It was found that treatment of FCS (50 mg) only in $\mathrm{HNO}_{3}$ or $\mathrm{H}_{2} \mathrm{SO}_{4}$ led to poor results with a large amount of remaining solid (Supplementary information, Table S1, entry 1 and 2), indicating a poor $Y$. At $\rho=1: 3$, all the FCS dissolved in the mixed acids regardless of $V_{\text {total }}$ after the same treatment and no large particles could be distinguished by the naked eye (entries 3-5). The same trend was observed for the samples with $\rho=1: 2$ (entries 6,7 ). When $\rho$ decreased to $1: 1$, high-quality dispersion could still be obtained at $V_{\text {total }}=12 \mathrm{~mL}$ (entry 8 ), while a small amount of solid was noticed at $V_{\text {total }}=4 \mathrm{~mL}$ (entry 9). The effect of the refluxing temperature on the quality of FCS dispersions was also examined. Specifically for the sample with $\rho=1: 1$ and $V_{\text {total }}=4 \mathrm{~mL}$, an increase in the refluxing temperature to $120^{\circ} \mathrm{C}$ led to the formation of a good dispersion (entry 10), while a decrease in the refluxing temperature to $80^{\circ} \mathrm{C}$ resulted in the appearance of a
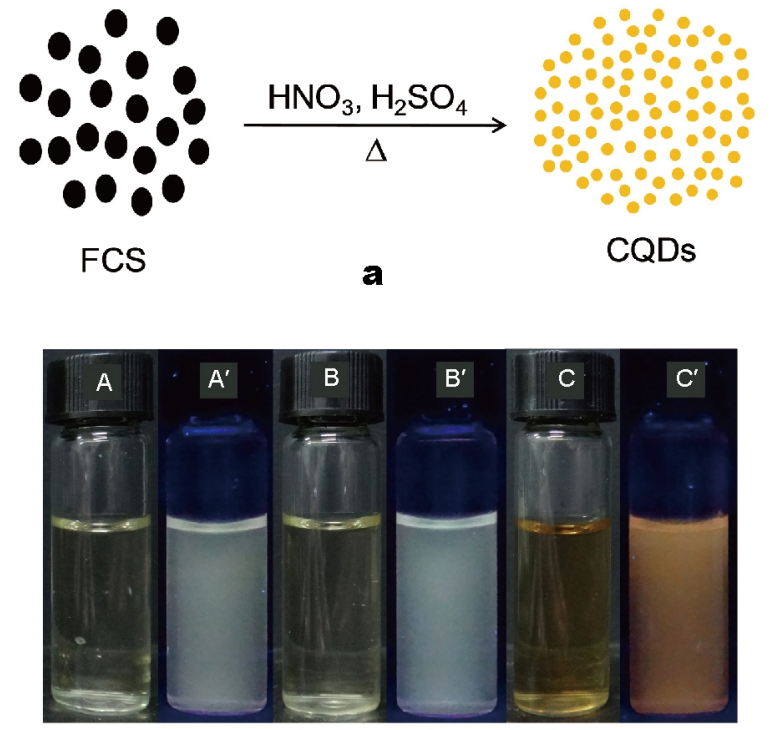

b

Figure 1 (a) Illustration of the synthesis of CQDs by acid-refluxing of FCS. (b) Photographs of CQDs in water obtained after successive dialysis of the sample obtained by refluxing $50 \mathrm{mg}$ FCS in mixed acids with $\rho=1: 1$ and $V_{\text {total }}=4 \mathrm{~mL}$. A. Concentrated dialysate between 500-1000 Da (sample II). B. Concentrated dialysate for the molecular cut-offs of 1000-2000 $\mathrm{Da}$ (sample III). C. The final product obtained after dialysis using a 2000 Da dialysis bag (sample IV). A'-C'. Photographs of samples II-IV when exposed to UV irradiation $(365 \mathrm{~nm})$.

large amount of solids (entry 11). The quality of dispersions was also affected by the refluxing time. When the refluxing time was decreased to $12 \mathrm{~h}$, some solid could still be observed at the bottom (entry 12). This could be totally eliminated by increasing the refluxing time to $36 \mathrm{~h}$ (entry 13).

The mixture obtained from acid refluxing was first diluted by deionized water and neutralized with $\mathrm{K}_{2} \mathrm{CO}_{3}$. After the removal of insoluble substances and large particles by centrifugation, the sample was subjected to dialysis to obtain CQD dispersions. This treatment results in a mean $Y$ of $40 \pm 5 \%$, which is much higher than that obtained from acid refluxing of CNTs (2\%) [20], coal (10-20\%) [23], candle soot (5-10\%) [29], and some biomasses such as bagasse (3\%) [40]. The as-prepared CQD dispersion contained particles with different sizes. In order to analyze the size distribution of CQD particles and to investigate its effect on the properties of the CQDs, a stepwise dialysis methodology was developed. For this purpose, the sample from entry 13 was used. Briefly, the filtrate was first dialyzed with a dialysis bag with a molecular cut-off of $100 \mathrm{Da}$. The dialysate 
which contained inorganic salts and tiny fragments was discarded, while the sample within the dialysis bag was transferred to another dialysis bag with a molecular cut-off of $500 \mathrm{Da}$. In this case, the dialysate was collected and concentrated to $\sim 5 \mathrm{~mL}$ for further characterizations and the sample within the dialysis bag was again transferred to another dialysis bag with a molecular cut-off of $1000 \mathrm{Da}$. The same procedure was repeated and a final dialysis was carried out using a dialysis bag of $2000 \mathrm{Da}$ and the final product $(\sim 15 \mathrm{~mL})$ was obtained. After this successive dialysis, totally four fragments were obtained including the samples with the sizes of 100-500 Da (I), 500-1000 Da (II), 1000-2000 Da (III), and $>2000 \mathrm{Da}$ (IV). Sample I did not exhibit photoluminescence (PL) under UV irradiation (365 $\mathrm{nm}$ ), samples II-IV were photoluminescent, with sample IV being the major product, as seen from its much heavier color compared to the other two samples (Fig. 1b).

\section{Structural characterizations}

The structural features of the CQDs obtained from FCS were investigated in detail by a variety of techniques. Emphasis was mainly put on the major product, i.e., sample IV. Fig. 2 shows the typical TEM images of FCS (dispersed in ethanol) and the CQDs prepared in this study. It can be seen that FCS contained spherical particles with a diameter of $40-50 \mathrm{~nm}$ (Fig. 2a). This size distribution is much narrower compared to that of candle soot $(20-800 \mathrm{~nm})$ [29] and is also quite different from that of coal. Anthracite contains particles with irregular size and shape, while coke particles are spherical in shape and have a diameter of $\sim 110$ $\mu \mathrm{m}$ [23]. The small size and regular shape of FCS particles make it an ideal candidate for the production of CQDs without ball-milling and account for the much higher $Y$ compared to other carbon materials. After refluxing in the mixed acids, the CQDs with a spherical shape and a diameter of 2-3 nm were obtained, as can be seen from Fig. $2 \mathrm{~b}$.

Figure 3 shows the Raman spectroscopy, XRD, TGA, FTIR spectroscopy, and XPS results of the CQDs (sample IV) and FCS. The two peaks at 1320-1340 (D band) and $1560-1590 \mathrm{~cm}^{-1}$ (G band) observed in the Raman spectra (Fig. 3a) correspond to the vibrations of carbon atoms with disordered carbon structure $\left(\mathrm{sp}^{3}\right)$ and $\mathrm{sp}^{2}$-bonded carbon atoms with a crystal lattice, respectively [41,42]. The intensity ratio of $\mathrm{D}$ and $\mathrm{G}$ bands $\left(I_{\mathrm{D}} / I_{\mathrm{G}}\right)$, is normally used as a measure of the defect-density in carbon-rich materials. For both FCS and the CQDs, the D band is more pronounced than the $\mathrm{G}$ band, indicating that both of them contain a large number of defect sites. The $I_{\mathrm{D}} / I_{\mathrm{G}}$ for FCS is calculated to be 1.317 , which is higher than that of the CQDs (1.064), indicating a higher $\mathrm{sp}^{2}$ content in the CQDs compared to that in FCS. The XRD patterns of both FCS and the CQDs (Fig. 3b) show a broad peak at $2 \theta=24^{\circ}$, which corresponds to a lattice distance of $3.4 \AA$. The presence of broad diffraction peaks shows that both FCS and the CQDs are amorphous. FTIR spectrum (Fig. 3c) of the CQDs shows a range of absorption bands at 3410, 2948, 1725,1600 , and $1420 \mathrm{~cm}^{-1}$ corresponding to $-\mathrm{OH}$ stretching, $\mathrm{C}-\mathrm{H}$ stretching, $\mathrm{C}=\mathrm{O}$ stretching, $\mathrm{C}=\mathrm{C}$ stretching, and $\mathrm{O}-\mathrm{H}$ bending vibrations, respectively. Compared to $\mathrm{FCS}$, the CQDs show higher intensity peaks for $-\mathrm{OH}$ and $\mathrm{C}=\mathrm{O}$ stretching vibrations, which is indicative of the successful introduction of hydroxyl and carboxy groups to the CQDs during acid-refluxing.

The change in the compositions of FCS before and after acid-refluxing is also evident from the TGA, XPS, and elemental analysis. The TGA results reveal that the weight loss in the CQDs is much more pronounced than that in FCS (Fig. 3d), indicating a much higher content of organic
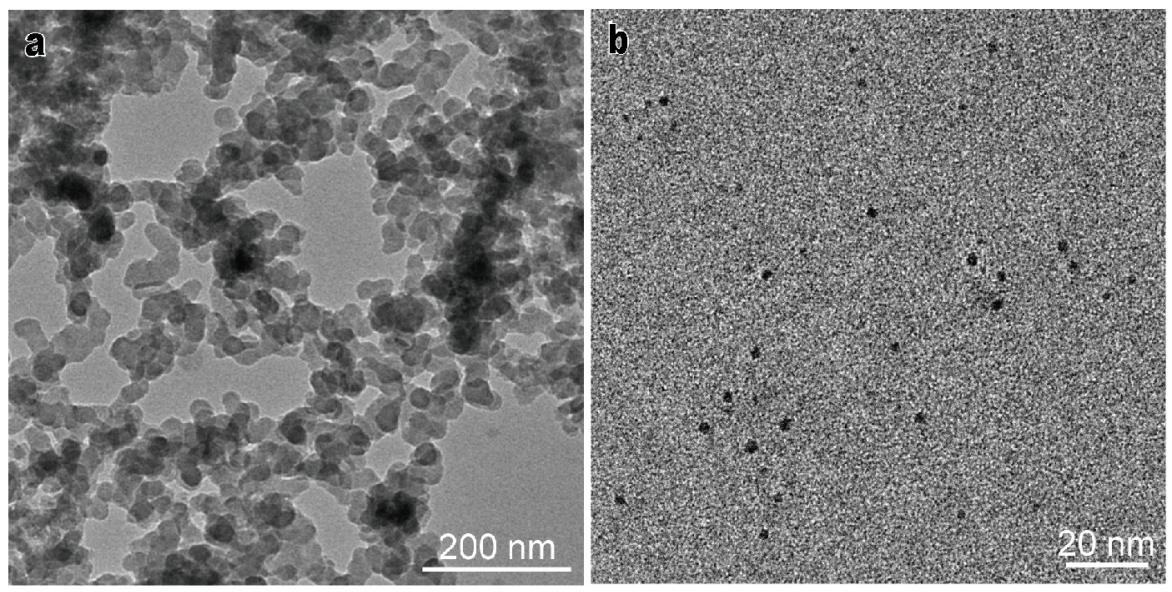

Figure 2 Typical TEM images of (a) FCS and (b) CQDs (sample IV). 

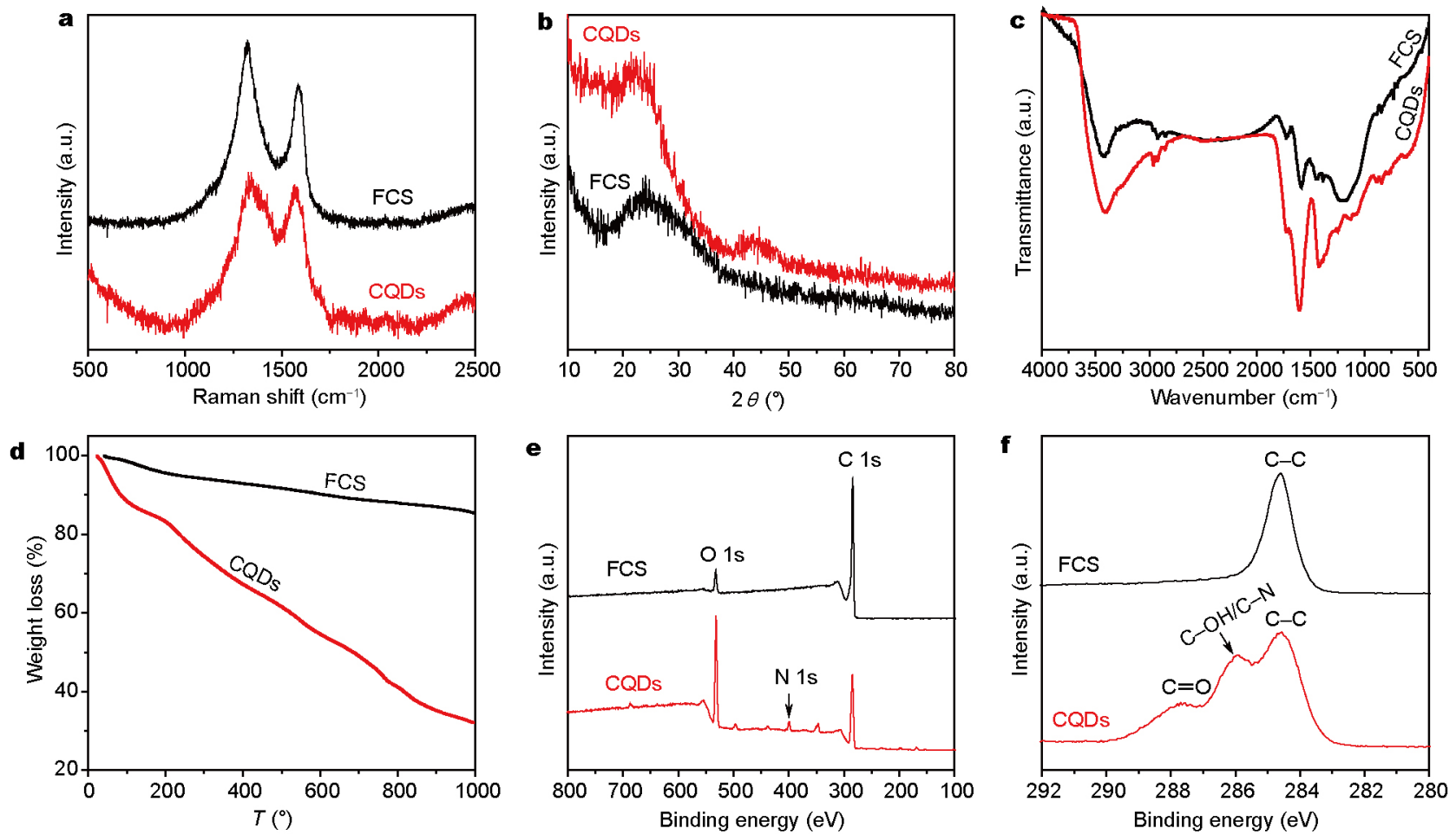

Figure 3 Characterization of CQDs (sample IV) and FCS. (a) Raman spectra, (b) XRD diffraction, (c) FTIR spectra, (d) TGA, (e) XPS survey and (f) magnification of the $\mathrm{C} 1 \mathrm{~s}$ peak.

Table 1 Elemental analysis results for FCS and CQDs

\begin{tabular}{ccccc}
\hline & $\mathrm{C}$ & $\mathrm{H}$ & $\mathrm{N}$ & $\mathrm{O}^{\mathrm{a}}$ \\
\hline $\mathrm{FCS}$ & $93.50 \%$ & $1.01 \%$ & $0.08 \%$ & $5.38 \%$ \\
CQDs & $36.74 \%$ & $2.37 \%$ & $0.73 \%$ & $59.67 \%$ \\
\hline
\end{tabular}

a) Calculated values.

functional groups. The XPS spectrum of FCS (Fig. 3e) reveals that it is mainly composed of carbon and oxygen. After the conversion to the CQDs, the carbon content of FCS decreases, while the oxygen content increases significantly. In addition, an $\mathrm{N}$ 1s signal also appears. The $\mathrm{C} 1 \mathrm{~s}$ spectra reveal that along with the $\mathrm{C}-\mathrm{C}(284.5 \mathrm{eV})$ peak, the CQDs also show the $\mathrm{C}-\mathrm{OH} / \mathrm{C}=\mathrm{N}(285.9 \mathrm{eV})$ and $\mathrm{C}=\mathrm{O}(287.8 \mathrm{eV})$ peaks, which are absent in the case of FCS. From the elemental analysis (Table 1) results, it can be seen clearly that the carbon content decreases greatly after acid refluxing, and a slight increase in the nitrogen content is also noticed. The calculated oxygen content of the CQDs produced in this study is $59.67 \%$, which is slightly higher than that of CQDs synthesized from CNTs (55\%) [20].

\section{Spectroscopic properties}

Most of the previous studies on CQDs have reported that
CQDs emit blue light, with an exception of a few studies reporting longer wavelength emissions (such as the emission of red light) [31,43-45]. Interestingly, the main product of the CQDs (sample IV) could emit yellow light, as evidenced from Fig. 1b. To fully reveal the spectroscopic properties of the CQDs, UV-vis, steady-state, and time-resolved fluorescence measurements were carried out. The results for sample IV are shown in Fig. 4. The UV-vis spectrum of the CQDs shows a broad absorption, which decreases continuously with an increase in the wavelength (Fig. 4a). The absorption obeys the Lambert-Beer rule within the investigated concentration range (inset of Fig. 4a), indicating that no self-assembly occurrs because of the high water solubility of the CQDs. Fig. 4b shows the emission of the CQDs under a 470-nm excitation at various concentrations. It is found that the PL intensity first increases and then decreases with a maximum at $0.5 \mathrm{mg} \mathrm{mL}^{-1}$. Meanwhile, a slight shift in the emission towards longer wavelengths is noticed. The effect of the excitation wavelength on the PL properties of the CQDs was also examined (Fig. $4 c, d)$. With an increase in the excitation wavelength, the PL intensity first increases to reach a maximum at $\lambda_{\text {ex }}=470 \mathrm{~nm}$ and then decreases. At $470 \mathrm{~nm} \leq \lambda_{\text {ex }} \leq 540 \mathrm{~nm}$, the emission peak continuously shifts from 527 to $571 \mathrm{~nm}$. This excita- 

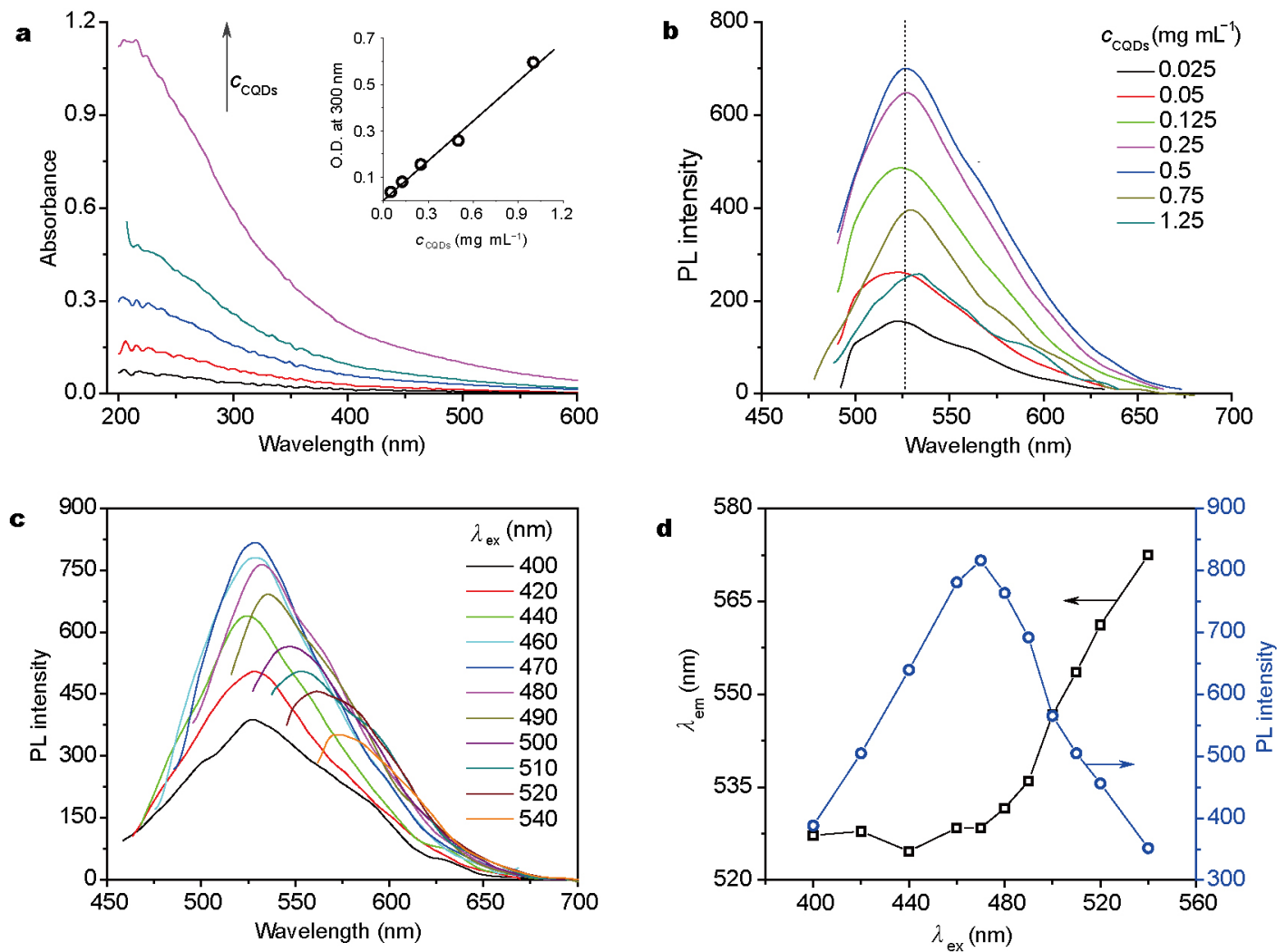

Figure 4 (a) UV-vis absorption of the CQD aqueous solutions (sample IV) with varying concentrations. Inset shows the absorbance at $300 \mathrm{~nm}$ as a function of the CQD concentration. (b) Concentration-dependent emission of the CQDs at an excitation wavelength $\left(\lambda_{\mathrm{ex}}\right)$ of $470 \mathrm{~nm}$. The dotted line is a guide for the eyes. (c) Emission at varying $\lambda_{\mathrm{ex}}$ of the CQDs in water $\left(0.667 \mathrm{mg} \mathrm{mL}^{-1}\right)$. (d) Variations of the emission wavelength $\left(\lambda_{\mathrm{em}}\right)$ and PL intensity as a function of $\lambda_{\mathrm{ex}}$.

tion-dependent PL behavior is similar to that observed for other luminescent carbon nanoparticles $[46,47]$. At $\lambda_{\text {ex }}<470 \mathrm{~nm}$, the position of the emission peak remains almost unchanged and an even blue-shift is noticed from 420 to $430 \mathrm{~nm}$. This trend is similar to the PL behavior of graphene quantum dots (GQDs) [48] and CQDs derived from CNTs [20]. The value $\Phi$ at the maximum emission, i.e., at $\lambda_{\mathrm{ex}}=470 \mathrm{~nm}$, is measured to be $3.13 \%$, which is either comparable to or higher than the previously reported values under similar treatments [20,22,29,30,49]. The PL properties of sample II and sample III were also investigated and the results are shown in Figs S1 and S2, respectively. A similar excitation-dependent PL behavior was obtained for both samples. However, the emission wavelengths are slightly lower than those of sample IV, which is consistent with the literature reports claiming that larger CQDs exhibit longer wavelength emissions $[47,50]$. The value of $\Phi$ for sample II is found to be $5.47 \%$, while that of sample III is $5.35 \%$. These values are higher than the value for sample IV. This is also consistent with the previous reports in which the CQDs with shorter-wavelength emissions or smaller sizes show higher $\Phi$ values $[51,52]$.

To get further information on the PL properties of the CQDs (sample IV), their fluorescence decay profile was measured. As shown in Fig. 5, a biexponential decay is observed and the values of $\tau_{1}$ and $\tau_{2}$ are found to be 0.88 (59.43\%) and $4.66 \mathrm{~ns}(40.57 \%)$, respectively, which gives an average value $(\langle\tau\rangle)$ of $2.41 \mathrm{~ns}$. This indicates that multiple radiative and non-radiative energy transfer pathways are involved in the emission of the CQDs. The biexponential decay of the CQDs and the excitation-dependent PL properties revealed by the steady-state fluorescence measurements (Fig. 4c) indicate that the PL properties of the CQDs are complicated. This is partially caused by the fact that the CQDs are a complex mixture of particles with different sizes and surface properties irrespective of the narrowing down of their size distributions by stepwise dialysis. More importantly, although significant progress has been achieved in the field of CQDs in the past decade, their PL mechanism remains a mystery and a universal explanation is still absent. Besides the mechanism based on the emission of $\pi$-conjugated moieties in the cores, it is generally 


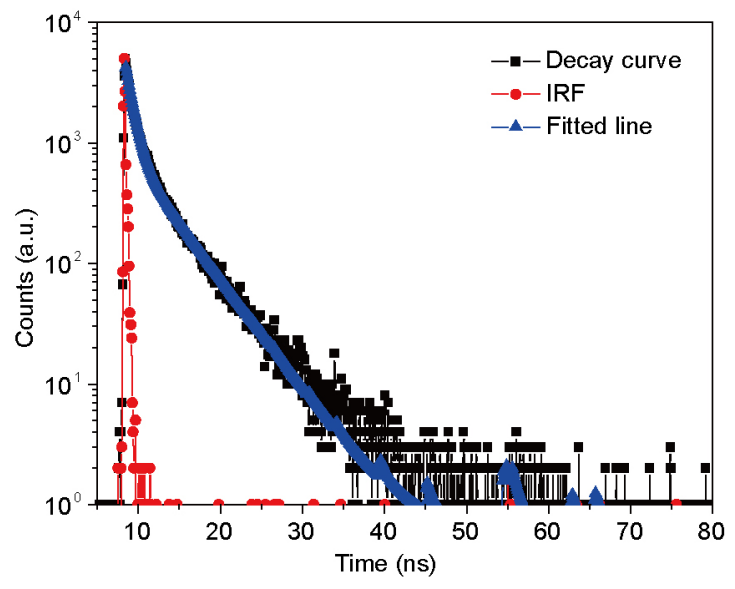

Figure 5 Fluorescence decay profile and fitted curve for the CQDs in water.

accepted that the PL properties of CQDs are governed by their surface states or defects caused by surface oxidation $[52,53]$, which serve as centers for capturing excitons and make CQDs photoluminescent. As our CQDs have a higher oxygen content compared to their counterparts prepared by other carbon nanomaterials, the number of capture centers on their surfaces is also larger, accounting for their yellow-emitting characteristics. Indeed, theories based on the highest occupied molecular orbit (HOMO)-lowest unoccupied molecular orbit (LUMO) gaps predict a red-shift of the PL with an increase in the degree of surface oxidation [44].

For practical applications, CQDs should exhibit stable PL properties. We stored the CQDs in water and noticed that they were very stable after 4 months and no precipitation or deterioration in their PL properties occurred. The $\mathrm{pH}$-dependence of the PL properties of the CQDs was checked
(Fig. 6a and Fig. S3). It was found that the PL intensity was stable within a $\mathrm{pH}$ range of $4-10$. In highly acidic $(\mathrm{pH}=$ 2 ) or alkaline $(\mathrm{pH}=12)$ solutions, an obvious decrease of the PL intensity was noticed. The emission wavelength first moves to higher values when the $\mathrm{pH}$ is increased from 2 to 8 , then decreases with a further increase in the $\mathrm{pH}$. Similar trend has also been reported previously [23,24,45,54-56], which could be caused by the change of surface charge density induced by the protonation-deprotonation processes.

Next, the stability of the CQDs in solutions with high salinity (induced by $\mathrm{NaCl}$ ) was examined. As seen from Fig. $6 \mathrm{~b}$ and Fig. S4, at an $\mathrm{NaCl}$ concentration $\left(c_{\mathrm{NaCl}}\right)$ of $0.2 \mathrm{~mol}$ $\mathrm{L}^{-1}$, the PL intensity is even higher compared to the original solution, after which the PL intensity remains almost constant up to $c_{\mathrm{NaCl}}=1.0 \mathrm{~mol} \mathrm{~L}^{-1}$. The exact reason for the initial increase in the PL intensity is not known currently. It is believed to be caused by the dehydration (induced by the addition) of the hydrophilic functional groups of the CQDs. Finally, the effect of UV irradiation at $365 \mathrm{~nm}(\mathrm{Hg}$ lamp, $220 \mathrm{~V}, 18 \mathrm{~W}$ ) on the PL properties of the CQDs was examined. The result shows that the PL intensity remains stable under UV irradiation for up to $12 \mathrm{~h}$ (Fig. S5). Hence, the PL stability of the CQDs is better than that of organic dyes and even some other CQDs undergoing photobleaching [57].

\section{CONCLUSIONS}

In summary, we demonstrated that FCS can be an ideal candidate for the production of CQDs. FCS has evenly-distributed particles with regular and narrow size distributions, leading to a high $Y$ after acid refluxing. As the source of FCS is sustainable and cheap, this synthetic route has the potential to be scaled up to realize industrial applications.
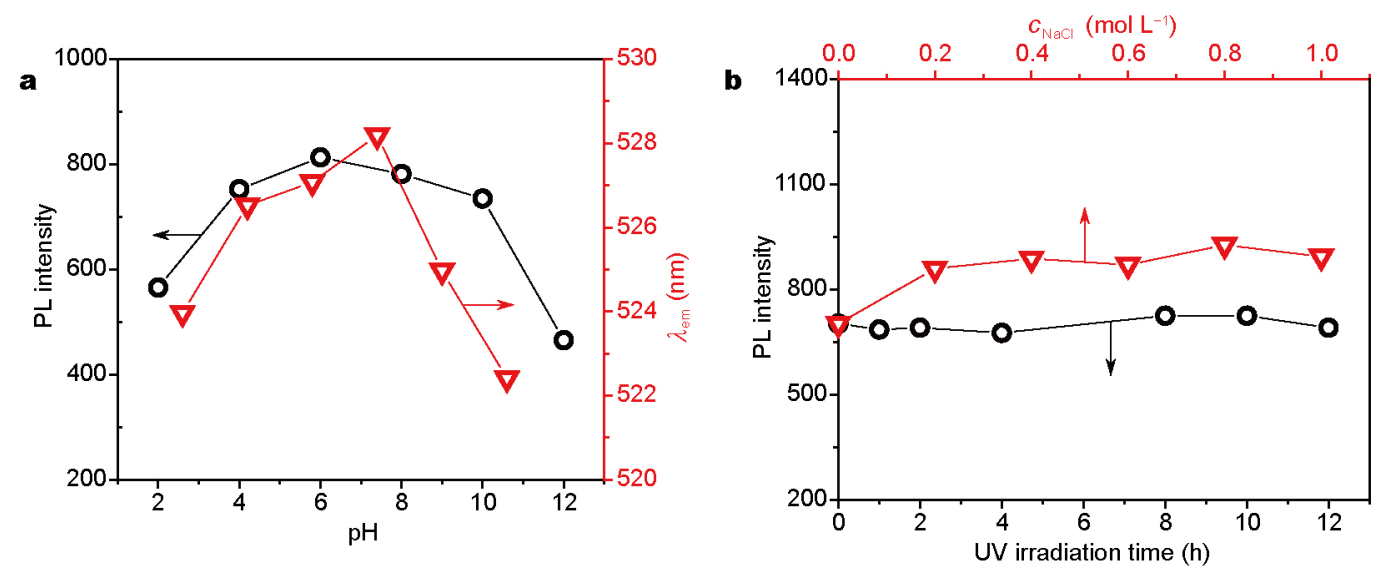

Figure 6 (a) Variations of $\lambda_{\mathrm{em}}$ and PL intensity of the CQDS as a function of $\mathrm{pH}$ and (b) variation of the PL intensity of the CQDs as a function of the $\mathrm{NaCl}$ concentration and $\mathrm{UV}$ irradiation time in water $\left(0.5 \mathrm{mg} \mathrm{mL}^{-1}\right)$. 
This is important to reduce the cost of CQDs and to expand their applications in various fields. The relatively high $\Phi$ and yellow-emitting characteristics of the as-obtained CQDs make them a promising fluorescent nanomaterial for a wide range of applications, especially in biology and life sciences. In addition, the high photostability of the CQDs in a wide range of $\mathrm{pH}$ and in harsh conditions with high salinity and long-term UV irradiation can further expand their applications.

Received 21 November 2016; accepted 26 December 2016; published online 16 January 2017

1 Alivisatos AP. Semiconductor clusters, nanocrystals, and quantum dots. Science, 1996, 271: 933-937

2 Michalet X, Pinaud FF, Bentolila LA, et al. Quantum dots for live cells, in vivo imaging, and diagnostics. Science, 2005, 307: 538-544

3 Resch-Genger U, Grabolle M, Cavaliere-Jaricot S, et al. Quantum dots versus organic dyes as fluorescent labels. Nat Meth, 2008, 5: 763-775

4 Lovric J, Cho SJ, Winnik FM, et al. Unmodified cadmium telluride quantum dots induce reactive oxygen species formation leading to multiple organelle damage and cell death. Chem Biol, 2005, 12: 1227-1234

5 Hardman R. A toxicologic review of quantum dots: toxicity depends on physicochemical and environmental factors. Environ Health Perspect, 2006, 114: 165-172

6 Geys J, Nemmar A, Verbeken E, et al. Acute toxicity and prothrombotic effects of quantum dots: impact of surface charge. Environ Health Perspect, 2008, 116: 1607-1613

$7 \mathrm{Xu}$ X, Ray R, Gu Y, et al. Electrophoretic analysis and purification of fluorescent single-walled carbon nanotube fragments. J Am Chem Soc, 2004, 126: 12736-12737

8 Baker SN, Baker GA. Luminescent carbon nanodots: emergent nanolights. Angew Chim Int Ed, 2010, 49: 6726-6744

9 Li H, Kang Z, Liu Y, et al. Carbon nanodots: synthesis, properties and applications. J Mater Chem, 2012, 22: 24230-24253

10 Lim SY, Shen W, Gao Z. Carbon quantum dots and their applications. Chem Soc Rev, 2015, 44: 362-381

11 Yu C, Liu Z, Chen Y, et al. CoS nanosheets-coupled graphene quantum dots architectures as a binder-free counter electrode for high-performance DSSCs. Sci China Mater, 2016, 59: 104-111

12 Sun YP, Zhou B, Lin Y, et al. Quantum-sized carbon dots for bright and colorful photoluminescence. J Am Chem Soc, 2006, 128: $7756-7757$

$13 \mathrm{Hu}$ SL, Niu KY, Sun J, et al. One-step synthesis of fluorescent carbon nanoparticles by laser irradiation. J Mater Chem, 2009, 19: 484-488

14 Li X, Wang H, Shimizu Y, et al. Preparation of carbon quantum dots with tunable photoluminescence by rapid laser passivation in ordinary organic solvents. Chem Commun, 2011, 47: 932-934

15 Wang J, Wang CF, Chen S. Amphiphilic egg-derived carbon dots: rapid plasma fabrication, pyrolysis process, and multicolor printing patterns. Angew Chem Int Ed, 2012, 51: 9297-9301

16 Zhou J, Booker C, Li R, et al. An electrochemical avenue to blue luminescent nanocrystals from multiwalled carbon nanotubes (MWCNTs). J Am Chem Soc, 2007, 129: 744-745

17 Zhao QL, Zhang ZL, Huang BH, et al. Facile preparation of low cytotoxicity fluorescent carbon nanocrystals by electrooxidation of graphite. Chem Commun, 2008, 5116
18 Lu J, Yang J, Wang J, et al. One-pot synthesis of fluorescent carbon nanoribbons, nanoparticles, and graphene by the exfoliation of graphite in ionic liquids. ACS Nano, 2009, 3: 2367-2375

19 Li H, Ming H, Liu Y, et al. Fluorescent carbon nanoparticles: electrochemical synthesis and their $\mathrm{pH}$ sensitive photoluminescence properties. New J Chem, 2011, 35: 2666-2670

20 Tao $\mathrm{H}$, Yang $\mathrm{K}, \mathrm{Ma} \mathrm{Z}$, et al. In vivo NIR fluorescence imaging, biodistribution, and toxicology of photoluminescent carbon dots produced from carbon nanotubes and graphite. Small, 2012, 8: 281-290

21 Peng J, Gao W, Gupta BK, et al. Graphene quantum dots derived from carbon fibers. Nano Lett, 2012, 12: 844-849

22 Qiao ZA, Wang Y, Gao Y, et al. Commercially activated carbon as the source for producing multicolor photoluminescent carbon dots by chemical oxidation. Chem Commun, 2009, 46: 8812-8814

23 Ye R, Xiang C, Lin J, et al. Coal as an abundant source of graphene quantum dots. Nat Commun, 2013, 4: 2943

$24 \mathrm{Hu} \mathrm{C}, \mathrm{Yu} \mathrm{C}, \mathrm{Li} \mathrm{M}$, et al. Chemically tailoring coal to fluorescent carbon dots with tuned size and their capacity for $\mathrm{Cu}$ (II) detection. Small, 2014, 10: 4926-4933

25 Dong Y, Lin J, Chen Y, et al. Graphene quantum dots, graphene oxide, carbon quantum dots and graphite nanocrystals in coals. Nanoscale, 2014, 6: 7410-7415

26 Wu M, Wang Y, Wu W, et al. Preparation of functionalized watersoluble photoluminescent carbon quantum dots from petroleum coke. Carbon, 2014, 78: 480-489

27 Wang Y, Wu W, Wu M, et al. Yellow-visual fluorescent carbon quantum dots from petroleum coke for the efficient detection of $\mathrm{Cu}^{2+}$ ions. New Carbon Mater, 2015, 30: 550-559

28 Shao X, Wu W, Wang R, et al. Engineering surface structure of petroleum-coke-derived carbon dots to enhance electron transfer for photooxidation. J Catal, 2016, 344: 236-241

29 Liu H, Ye T, Mao C. Fluorescent carbon nanoparticles derived from candle soot. Angew Chem Int Ed, 2007, 46: 6473-6475

30 Tian L, Ghosh D, Chen W, et al. Nanosized carbon particles from natural gas soot. Chem Mater, 2009, 21: 2803-2809

31 Ko HY, Chang YW, Paramasivam G, et al. In vivo imaging of tumour bearing near-infrared fluorescence-emitting carbon nanodots derived from tire soot. Chem Commun, 2013, 49: 10290-10292

32 Bourlinos AB, Stassinopoulos A, Anglos D, et al. Photoluminescent carbogenic dots. Chem Mater, 2008, 20: 4539-4541

33 Bourlinos AB, Stassinopoulos A, Anglos D, et al. Surface functionalized carbogenic quantum dots. Small, 2008, 4: 455-458

34 Krysmann MJ, Kelarakis A, Dallas P, et al. Formation mechanism of carbogenic nanoparticles with dual photoluminescence emission. J Am Chem Soc, 2012, 134: 747-750

35 Zhai X, Zhang P, Liu C, et al. Highly luminescent carbon nanodots by microwave-assisted pyrolysis. Chem Commun, 2012, 48: 7955-7957

36 Egashira M, Okada S, Korai Y, et al. Toluene-insoluble fraction of fullerene-soot as the electrode of a double-layer capacitor. J Power Sources, 2005, 148: 116-120

37 Spassova I, Khristova M, Nickolov R, et al. Novel application of depleted fullerene soot (DFS) as support of catalysts for low-temperature reduction of NO with CO. J Colloid Interface Sci, 2008, 320: 186-193

$38 \mathrm{Hu} \mathrm{C}, \mathrm{Yu} \mathrm{C}, \mathrm{Li} \mathrm{M}$, et al. Preparation of single-walled carbon nanotubes from fullerene waste soot. ACS Sustainable Chem Eng, 2014, 2: 14-18

39 Ciesielski A, Haar S, Aliprandi A, et al. Modifying the size of ultrasound-induced liquid-phase exfoliated graphene: from nanosheets 
to nanodots. ACS Nano, 2016, 10: 10768-10777

40 Jiang BP, Zhou B, Shen XC, et al. Selective probing of gaseous ammonia using red-emitting carbon dots based on an interfacial response mechanism. Chem Eur J, 2015, 21: 18993-18999

41 Chen X, Lee GS, Zettl A, et al. Biomimetic engineering of carbon nanotubes by using cell surface mucin mimics. Angew Chem Int Ed, 2004, 43: 6111-6116

42 Xin $\mathrm{X}, \mathrm{Xu} \mathrm{G}, \mathrm{Zhao} \mathrm{T}$, et al. Dispersing carbon nanotubes in aqueous solutions by a starlike block copolymer. J Phys Chem C, 2008, 112: $16377-16384$

43 Jiang K, Sun S, Zhang L, et al. Red, green, and blue luminescence by carbon dots: full-color emission tuning and multicolor cellular imaging. Angew Chem Int Ed, 2015, 54: 5360-5363

$44 \mathrm{Hu}$ S, Trinchi A, Atkin P, et al. Tunable photoluminescence across the entire visible spectrum from carbon dots excited by white light. Angew Chem Int Ed, 2015, 54: 2970-2974

45 Ding H, Yu SB, Wei JS, et al. Full-color light-emitting carbon dots with a surface-state-controlled luminescence mechanism. ACS Nano, 2016, 10: 484-491

46 Pan D, Zhang J, Li Z, et al. Observation of pH-, solvent-, spin-, and excitation-dependent blue photoluminescence from carbon nanoparticles. Chem Commun, 2010, 46: 3681-3683

$47 \mathrm{Li} \mathrm{H}, \mathrm{He} \mathrm{X}$, Kang Z, et al. Water-soluble fluorescent carbon quantum dots and photocatalyst design. Angew Chim Int Ed, 2010, 49: 4430-4434

48 Shen J, Zhu Y, Yang X, et al. One-pot hydrothermal synthesis of graphene quantum dots surface-passivated by polyethylene glycol and their photoelectric conversion under near-infrared light. New J Chem, 2012, 36: 97-101

49 Peng H, Travas-Sejdic J. Simple aqueous solution route to luminescent carbogenic dots from carbohydrates. Chem Mater, 2009, 21 : 5563-5565

50 Li L, Wu G, Yang G, et al. Focusing on luminescent graphene quantum dots: current status and future perspectives. Nanoscale, 2013, 5: 4015-4039
51 Fan J, Chu PK. Group IV nanoparticles: synthesis, properties, and biological applications. Small, 2010, 6: 2080-2098

52 Bao L, Liu C, Zhang ZL, et al. Photoluminescence-tunable carbon nanodots: surface-state energy-gap tuning. Adv Mater, 2015, 27: 1663-1667

53 Wang L, Zhu SJ, Wang HY, et al. Common origin of green luminescence in carbon nanodots and graphene quantum dots. ACS Nano, 2014, 8: 2541-2547

54 Dong Y, Pang H, Yang HB, et al. Carbon-based dots co-doped with nitrogen and sulfur for high quantum yield and excitation-independent emission. Angew Chem Int Ed, 2013, 52: 7800-7804

55 Song Y, Zhu S, Xiang S, et al. Investigation into the fluorescence quenching behaviors and applications of carbon dots. Nanoscale, 2014, 6: 4676-4682

56 Wu ZL, Gao MX, Wang TT, et al. A general quantitative $\mathrm{pH}$ sensor developed with dicyandiamide $\mathrm{N}$-doped high quantum yield graphene quantum dots. Nanoscale, 2014, 6: 3868-3874

57 Zhu S, Meng Q, Wang L, et al. Highly photoluminescent carbon dots for multicolor patterning, sensors, and bioimaging. Angew Chem Int Ed, 2013, 52: 3953-3957

Acknowledgments This work was supported by the National Natural Science Foundation of China (21402215 and 61474124) and the Hundred Talents Program of the Chinese Academy of Sciences (Y20245YBR1).

Author contributions Zhang Q, Sun X, and Ruan H prepared the CQDs from FCS and performed most of the characterizations of the CQDs. Yin K contributed to the FTIR and XPS measurements of the CQDs. Li H designed the project, discussed the results, and wrote the paper.

Conflict of interest The authors declare that they have no conflict of interest.

Supplementary information Additional fluorescence spectra and details of the preparation of CQDs are available in the online version of the paper. 


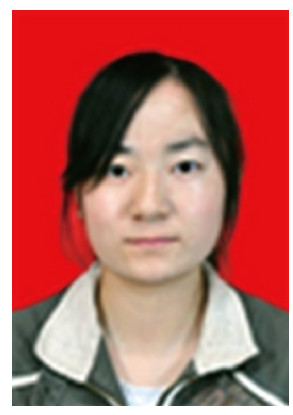

Qinghong Zhang was born in 1992. She obtained her BSc degree in chemistry from North University of China in 2015 and is currently a graduate student at China Research Institute of Daily Chemical Industry. She is now involved in the synthesis and applications of surfactants.

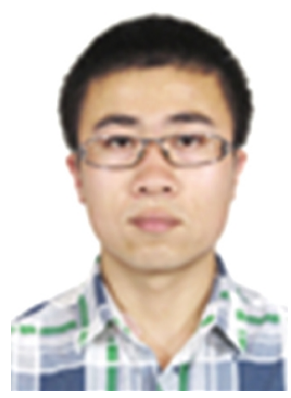

Xiaofeng Sun was born in 1989. He obtained his MSc degree in applied chemistry from China Research Institute of Daily Chemical Industry in 2015 and is currently a PhD candidate at Lanzhou Institute of Chemical Physics, Chinese Academy of Sciences under the supervision of Prof. Hongguang Li. His research is mainly focused on the preparation and applications of carbon quantum dots.

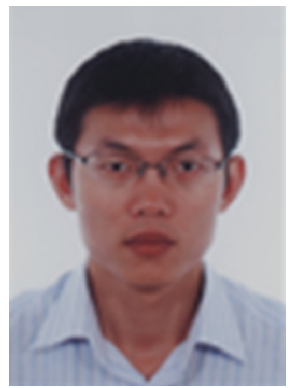

Hongguang Li was born in 1979. He obtained his BSc degree and PhD degree from Shandong University in 2003 and 2008, respectively. After three years of postdoctoral research, he joined the Laboratory of Clean Energy Chemistry and Materials, Lanzhou Institute of Chemical Physics, Chinese Academy of Sciences as a full professor. His current research interest includes the design of novel $\pi$-conjugated molecules and engineering carbon nanomaterials such as fullerenes and carbon quantum dots for applications in optoelectronic devices.

\section{利用富勒烯炭灰制备黄光发射碳量子点}

张庆红 ${ }^{1,2}$, 孙晓峰 ${ }^{1,3}$, 阮红 ${ }^{1}$, 尹 克样 $^{1}$, 李洪光 ${ }^{1 *}$

摘要 碳量子点已经成为具有巨大应用潜力的新一代光致发光纳米材料. 在碳量子点的众多制备方法中, 归属于“自上而下”法的酸回流处 理含碳块体材料的技术有望实现碳量子点的宏量制备. 其中, 来源丰富、价格低廉原材料的选择是关键. 本文中, 我们详细考察了以富勒烯 工业化生产过程中副产的炭灰为原料制备碳量子点的实际应用潜力. 研究发现, 在浓硝酸和浓硫酸作用下, 富勒烯炭灰能够成功地转化为 碳量子点. 与用其他原料通过类似方法制备的碳量子点相比, 富勒烯炭灰制备的碳量子点具有较高的菼光量子产率, 达 $3 \%-5 \%$. 更为重要的 是, 光激发下富勒烯炭灰制备的碳量子点能够发射黄色苂光, 从而在生命科学等领域具有潜在应用. 进一步研究表明, 富勒烯炭灰制备的碳 量子点在很宽的 $\mathrm{pH}$ 值范围内具有稳定的光致发光行为, 且能够抵御高的离子强度和长时间光照, 进而拓宽了其在苛刻条件下的应用潜力. 